Cahiers $d u$ MONDE RUSSE

\section{Cahiers du monde russe}

Russie - Empire russe - Union soviétique et États indépendants

$62 / 4 \mid 2021$

Varia

\title{
Natalya SOLOPOVA, La préfabrication en URSS. Concepts techniques et dispositifs architecturaux
}

\section{Fabien Bellat}

\section{OpenEdition \\ Journals}

\section{Édition électronique}

URL : https://journals.openedition.org/monderusse/12927

DOI : 10.4000/monderusse. 12927

ISSN : $1777-5388$

\section{Éditeur}

Éditions de l'EHESS

\section{Édition imprimée}

Date de publication : 1 décembre 2021

Pagination : 751-753

ISBN : 978-2-7132-2895-7

ISSN : $1252-6576$

\section{Référence électronique}

Fabien Bellat, «Natalya SOLOPOVA, La préfabrication en URSS. Concepts techniques et dispositifs architecturaux », Cahiers du monde russe [En ligne], 62/4 | 2021, mis en ligne le 01 décembre 2021 consulté le 03 septembre 2022. URL : http://journals.openedition.org/monderusse/12927 ; DOI https://doi.org/10.4000/monderusse. 12927

Ce document a été généré automatiquement le 3 septembre 2022.

Tous droits réservés 


\title{
Natalya SOLOPOVA, La préfabrication en URSS. Concepts techniques et dispositifs architecturaux
}

\author{
Fabien Bellat
}

\section{RÉFÉRENCE}

Natalya SOLOPOVA, La préfabrication en URSS. Concepts techniques et dispositifs

architecturaux, Berlin : Dom Publishers, (Coll. Histoire et Théorie, vol. 109) 2021, 192 p.

1 Le livre de Natalya Solopova reproduit intégralement sa thèse, soutenue en 2021 à l'université Paris 8, sous la direction conjointe de Jean-Louis Cohen et du regretté Yannis Tsiomis. Un bref avant-propos actualisé resitue de manière intéressante le délicat contexte de la recherche effectuée plus de vingt ans auparavant, mais ne cite que très partiellement quelques nouveaux travaux sur ce sujet essentiel dans l'histoire technique de l'architecture.

2 Après cette ouverture, l'introduction résume de manière générale le sujet, esquissant des parallèles rapides entre les situations française et soviétique. L'ouvrage comporte ensuite cinq chapitres généraux, bien synthétiques, suivis d'une conclusion globale, et d'appendices. Ceux-ci traitent de la normalisation de l'habitat, des instances administratives soviétiques gérant l'attribution des logements, des instituts de projets ou des entreprises de construction en URSS, et enfin un récapitulatif des administrations soviétiques du milieu de la construction. Disons d'emblée que ces très utiles et bien pensés appendices auraient gagné à être présentés au début du livre, afin de mieux faire comprendre au lecteur français les spécificités de l'URSS quant à l'habitat de masse. Ensuite, les annexes finales comportent des biographies de quelques architectes cités, rédigées dans la lignée de l'Encyclopédie soviétique, qui listait les titres et réalisations sans vraiment replacer ces auteurs dans leur contexte temporel et créatif. 
3 Le premier chapitre, titré « Modèles et modes d'organisation de la préfabrication » est très large dans ses ambitions, resituant l'effervescence intellectuelle de l'URSS des années 1920. Toutefois, le rappel de l'impact du taylorisme ou du fordisme chez les Soviétiques reste plutôt confus. Jean-Louis Cohen a d'ailleurs fait récemment avec Construire un nouveau Nouveau monde : l'amerikanizm dans l'architecture russe une synthèse magistrale revenant entre autres sur cette thématique. Une affirmation (p.22) selon laquelle l'URSS ne produisit que des usines fixes de préfabrication et ne recourut jamais à des unités mobiles reste assez problématique. En effet, lors de l'édification des ultimes villes nouvelles des années 1960-1970, comme Togliatti, il y eut bien des usines mobiles pour fabriquer in situ des panneaux, assemblés illico sur les proches chantiers.

Le deuxième chapitre, intitulé « Idéologie et modélisation des formes architecturales en URSS 1920-1950 » s'avère bien plus convaincant dans le choix de ses exemples et son argumentation. Les travaux pionniers de Viktor Vesnin et Andrej Burov dans les décennies 1930-1940 étaient déjà bien connus (cités par exemple par Anatole Kopp dans L'architecture de la période stalinienne, 1978), mais l'auteur en livre un commentaire technique efficace et publie quelques illustrations nouvelles. Les exemples aussi cités p. 50 à Magnitogorsk, élevés peu après la seconde guerre mondiale, contribuent bien à affiner la connaissance sur les hésitations entre préfabrication intégrale des bâtiments et adaptation à un vocabulaire esthétique traditionnel. De même, les fines observations de Solopova sur la capacité d'Ivan Žoltovskij - architecte passionné par la Renaissance et Palladio - à conjuguer conception formelle académique et modernisation technologique, sont parmi les passages les plus intéressants de cette publication.

Le troisième chapitre «La politique de l'architecture et de la construction de Khrouchtchev - ouverture vers un modèle nouveau 1954-1958» revient de manière instructive sur les circonstances qui ont poussé le nouveau maître du Kremlin à entamer la déstalinisation en prenant les architectes pour premiers boucs émissaires. Toutefois, peut-être l'auteure accorde-t-elle trop d'influence au virulent rapport que le jeune architecte Georgij Gradov avait envoyé à Hruščev, pour dénoncer les mandarins de l'architecture stalinienne. Sans doute le courrier de Gradov eut-il en effet un rôle déclencheur, mais le vibrionnant successeur de Stalin fonda aussi ses philippiques contre Aleksandr Vlassov ou Arkadij Mordvinov du fait de ses propres exaspérations face à des bâtisseurs, qui avaient travaillé sous son autorité, et ayant comme lui bâti leur carrière par la manipulation de la paranoïa stalinienne.

6 Le quatrième chapitre, «L'expérience française: une référence pour la préfabrication soviétique » commence par un résumé des choix français d'industrialisation du bâtiment après le second conflit mondial, et dévie ensuite vers les transferts de technologie entre les deux pays. Là, le rappel des intervenants s'avère instructif, citant notamment le voyage en URSS des architectes français Robert Camelot (un des auteurs du CNIT à La Défense), Guy Pison et Jacques Tournant (qui participèrent à la reconstruction du Havre) ou les entreprises spécialisées dans l'industrialisation du bâtiment, comme Coignet ou celle de l'ingénieur Raymond Camus. Visite intéressée, qui valut en retour aux Français la visite d'émissaires russes, dont Georgij Gradov. Si Solopova ne le cite pas, le pourtant disgracié Vlassov vint lui aussi examiner sur place les travaux français. Selon Solopova, Camus aurait bénéficié "d'une autorité certaine » (p.107) en URSS, qui l'aurait aidé à vendre ses brevets, pour créer deux usines à Taškent et Baku. Pour ma propre thèse (soutenue en 2007) je m'étais aussi intéressé à la question, sans parvenir à des résultats plus avancés que ceux rassemblés par Natalya Solopova. En fait, une réponse claire ne fut 
trouvée qu'en 2013, lorsque je menais à mon tour à Moscou des entretiens d'abord auprès de la famille de l'architecte Boris Rubanenko, puis avec ses anciens assistants et désormais directeurs à l'Institut central de recherche et de design des bâtiments résidentiels et publics. Tous pointèrent railleusement que la coopération avec Camus releva du circonscrit échantillon, pour tester à échelle 1 le potentiel des solutions du bâtisseur français. Aussi, le test des deux usines issues de conceptions françaises, situées de surcroît dans des républiques périphériques, tandis que l'URSS avait alors des milliers de sites de préfabrication de panneaux de béton, ne semble guère concluant...

Ce chapitre comporte par ailleurs une autre affirmation, celle-ci vraiment problématique (p.95) comme quoi en URSS seuls les immeubles collectifs auraient fait l'objet d'expérimentations, alors que la France aurait également mené des expériences sur les maisons individuelles ou groupées. Or, durant la seconde guerre mondiale, les Soviétiques se posèrent eux aussi la question d'un habitat de petite échelle. Un académique comme Žoltovskij étudia des maisons jumelées sur plans-types pour la reconstruction des villes dévastées, tandis que Burov et Nikolaj Kolli (deux ex-modernes convertis à la culture stalinienne par survie professionnelle) mirent à profit leur évacuation en Asie centrale pour dessiner des projets de bungalows adaptés au torride climat de ces régions, et standardisables soit avec des matériaux usinés soit avec des éléments vernaculaires. Hélas non réalisées, ces hypothèses alimentèrent la réflexion des instituts staliniens de projets spécialisés, qui diffusèrent largement des types de petits habitats à travers toute l'Union.

8 Le cinquième chapitre « La mise en place de la préfabrication industrielle à l'échelle de l'URSS " développe de précis descriptifs techniques de quelques-unes des séries d'immeubles préfabriqués, mais parvient néanmoins à modérer cette lecture potentiellement rébarbative par le choix habile de quelques caricatures du journal satirique Krokodil, moquant avec une douce ironie (dans les limites de ce qui était permis par le régime !) les défauts d'une architecture ultra-standardisée, imposant des cadres de vie monotones et contraignants.

9 Toutefois, dans ce chapitre comme dans le reste de l'ouvrage, certaines lacunes sont plutôt à regretter. D'abord, les évolutions technologiques des années 1970 sont largement passées sous silence. Pourtant, Philip Meuser, éditeur de cet ouvrage, a publié en 2015 avec Dimtri Zadorin dans Towards a Typology of Soviet Mass Housing, Prefabrication in the USSR 1955-1991 une synthèse très complète, détaillant avec une profusion de détails le potentiel ou les limites de chaque série, et les raisons de son maintien ou de son remplacement. Chacun à leur manière, les ouvrages de Solopova et de Meuser cernent un vaste sujet, offrant au lecteur de quoi évaluer les politiques soviétiques pour plus vite loger ses populations. Solopova se concentre sur des exemples russes, ne reproduisant qu'un ensemble lituanien, et cite sans les montrer des projets ukrainiens et biélorusses. Voici là un des gros manques de ce pourtant si utile ouvrage : la totale absence des séries faites en Ouzbékistan et au Tadjikistan, spécialement étudiées selon des solutions antisismiques (une vague allusion est faite au problème sismique p. 128, sans analyse des réponses géographiques spécifiques). Ensuite, de son propre aveu (p.147) Solopova ne traite pas la forme urbaine de ces grands ensembles: son travail permet donc de comprendre les conditions technologiques de l'industrialisation soviétique du bâtiment, mais reste hélas muet sur leur application spatiale. C'est regrettable, car évoquer la technique en la séparant de son environnement final aboutit à un texte paraissant 
suspendu dans les airs, comme une carcasse de béton accrochée à une grue, attendant de trouver sa place dans son édifice urbain.

10 Malgré ces quelques limites, causées surtout par le caractère pionnier de cette recherche, cet ouvrage demeure fort appréciable pour le lectorat français. Il complète à point nommé les récentes recherches russophones, anglophones et germanophones, apportant une synthèse nécessaire sur le développement d'une industrie de la construction qui a tant marqué le $\mathrm{xx}^{\mathrm{e}}$ siècle.

\section{AUTEURS}

\section{FABIEN BELLAT}

EVCAU, ENSA Paris Val de Seine 